\title{
BMJ Open Improving the well-being of men by Evaluating and Addressing the Gastrointestinal Late Effects (EAGLE) of radical treatment for prostate cancer: study protocol for a mixed-method implementation project
}

\author{
Sophia Taylor, ${ }^{1}$ Weyinmi Demeyin, ${ }^{2}$ Ann Muls, ${ }^{3}$ Catherine Ferguson, ${ }^{4}$ \\ Damian J J Farnell, ${ }^{5}$ David Cohen, ${ }^{6}$ Jervoise Andreyev, ${ }^{3}$ John Green, ${ }^{7}$ \\ Lesley Smith, ${ }^{8}$ Sam Ahmedzai, ${ }^{9}$ Sara Pickett, ${ }^{10}$ Annmarie Nelson, ${ }^{1}$ John Staffurth ${ }^{11,12}$
}

To cite: Taylor S,

Demeyin W, Muls A, et al. Improving the well-being of men by Evaluating and Addressing the

Gastrointestinal Late Effects (EAGLE) of radical treatment for prostate cancer: study protocol for a mixed-method implementation project. BMJ Open 2016;6: 011773.

doi:10.1136/bmjopen-2016011773

- Prepublication history and additional material is available. To view please visit the journal (http://dx.doi.org/ 10.1136/bmjopen-2016011773).

Received 3 March 2016

Revised 13 May 2016

Accepted 20 June 2016

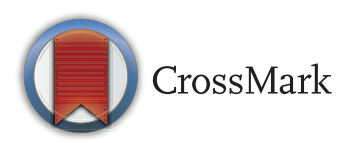

For numbered affiliations see end of article.

Correspondence to Dr Annmarie Nelson; nelsona9@cardiff.a.c.uk

\section{ABSTRACT}

Introduction: Radiotherapy treatment for prostate cancer can cause bowel problems, which may lead to severe difficulties for cancer survivors including limiting travel, work or socialising. These symptoms can appear at any time following radiotherapy. This study focuses on the early identification and protocol-based management of effects known to cause long-term, or even permanent, changes to the well-being of prostate cancer survivors. The rationale of this study is to improve the care offered to men and their families following pelvic radiotherapy for prostate cancer.

Method and analysis: Implementation research methodology will be used to adopt a multicomponent intervention at three UK centres. The intervention package comprises a standardised clinical assessment of relevant symptoms in oncology outpatient clinics and rapid referral to an enhanced gastroenterological service for patients identified with bowel problems. Gastroenterology staff will be trained to use an expertpractice algorithm of targeted gastroenterology investigations and treatments. The evaluation of the intervention and its embedding within local practices will be conducted using a mixed-methods design. The effect of the new service will be measured in terms of the following outcomes: acceptability to staff and patients; quality of life; symptom control and costeffectiveness. Data collection will take place at baseline, 6 months ( \pm 2 months), and 12 months ( \pm 2 months) after entry into the study.

Ethics and dissemination: The study has ethical approval from the North West-Liverpool East Research Ethics Committee and the appropriate NHS governance clearance. All participants provide written informed consent. The study team aim to publish the results of the study in peer-reviewed journals as well as at national and international conferences.

Trial registration number: UKCRN16974

\section{Strengths and limitations of this study}

- The study uses mixed methods, using both qualitative and quantitative approaches to data collection and analysis enabling solutions to any problems that arise can be made in real time.

- The study is restricted to the recruitment of patients with prostate cancer with late gastrointestinal effects of pelvic radiotherapy, thereby reducing the sample size and generalisability to other cancer diagnoses and treatment modalities. In addition, the implementation of the intervention may not be successful.

\section{INTRODUCTION}

Prostate cancer is the most common cancer in men in the UK except for nonmelanomatous skin cancer. Indeed, 47300 men were diagnosed in the UK with prostate cancer in 2013. ${ }^{1}$ Men with organ-confined localised disease have extremely good shortterm prostate cancer survival rates with over $95 \%$ alive at 5 years after diagnosis and $84 \%$ at 10 years. ${ }^{12}$ There are several radical treatment options available for prostate cancer. Much evidence exists relating to the shortterm and long-term effects of the major established therapies (prostatectomy, external beam radiotherapy and low-dose rate brachytherapy). ${ }^{3}$ Despite these side effects of treatment, many men remain satisfied with their treatment and they would select the same treatment again. ${ }^{4}$

Late effects of pelvic radiotherapy

Radiotherapy is important in the treatment of cancers. ${ }^{5}$ It is estimated conservatively that 
17000 patients with cancer receive pelvic radiotherapy per year in the UK. ${ }^{6}$ Of these an estimated $90 \%$ develop a permanent change in their bowel habit and 50\% experience a reduction in their quality of life, and 20$40 \%$ rate the effect on quality of life as moderate or severe. ${ }^{7}$ In a more recent study $35 \%$ of prostate patients with cancer suffered from bowel urgency 15 years after radiotherapy. ${ }^{8}$

Despite the significant impact of these symptoms on quality of life, only one in five patients who develop gastrointestinal (GI) symptoms after pelvic radiotherapy are referred to a gastroenterologist. ${ }^{9}$ There are several reasons for this low referral rate. First, patients may not offer up their symptoms to the treating doctor, despite the potentially catastrophic effects to their physical, emotional and social well-being. Explanations for this behaviour include reasons such as: patients may view them as expected consequences of therapy; they may be too embarrassed to mention them; or, they might just be grateful for being cured. ${ }^{10}$ Second, clinicians looking after patients might not seek information on late radiation effects, focusing primarily on symptoms of cancer, partly as late effects have been considered to be irreversible. $^{11}$

\section{Current patterns of care}

The long-term care of men with curable or cured prostate cancer varies between countries. There are also intracountry variations, particularly in the UK where healthcare has been devolved. ${ }^{12}$ Variations in managing late GI effects of pelvic radiotherapy across the UK have been highlighted by two recent surveys of patterns of care aimed at clinical oncologists and gastroenterologists. ${ }^{6}{ }^{13}$ The oncologist survey reported that 91 of 190 (48\%) responding clinicians refer $<10 \%$ of patients for specialist evaluation. ${ }^{6}$ The majority of responding oncologists $(58 \%)$ did not have a local or regional gastroenterological or colorectal surgical colleague with a special interest in postradiation bowel dysfunction and $52 \%$ of these oncologists felt their current service was inadequate. ${ }^{6}$ The results from the gastroenterologist survey were broadly similar. Most gastroenterologists (59\%) thought the service should be managed by a gastroenterologist with a special interest. However, most gastroenterologists $(81 \%)$ lacked a specialist interest, with only $48 \%$ rating their current service as adequate. ${ }^{13}$

\section{Managing late GI effects of pelvic radiotherapy}

Research carried out by the Gastro Intestinal and Nutrition Team (GIANT) service at The Royal Marsden National Health Service (NHS) Foundation Trust identified over 20 symptoms that occur frequently after prostate radiotherapy. ${ }^{14}$ Of the patients experiencing GI symptoms one-third will have a diagnosis unrelated to their radiotherapy. ${ }^{7}$ As the underlying diagnosis, symptoms and pathophysiology varies between patients, empirical therapies are unlikely to be successful. ${ }^{10}$
The Optimising Radiotherapy Bowel Injury Therapy (ORBIT) trial, ${ }^{9}$ demonstrated that these symptoms can be diagnosed accurately and that they can be treated effectively at very modest cost compared to the cost of the cancer treatment itself, and that this approach leads to improvements in pelvic radiation-induced GI symptoms as measured by Inflammatory Bowel Disease Questionnaire- Bowel (IBDQ-B) scores. The ORBIT trial showed that patients who were offered targeted interventions from clinicians who followed a detailed algorithm had better improvements in GI symptoms when compared to patients who were given usual care. ${ }^{9}$ This evidence also demonstrated that for most patients a specially trained nurse could deliver this assessment and treatment just as effectively as a consultant.

\section{Research required}

The Royal Marsden Hospital (RMH) is currently the only centre of excellence in the UK for managing late effects using the algorithm from the ORBIT trial. ${ }^{9}$ An implementation study is thus required to establish a new service to help manage the late GI effects that develop after pelvic radiotherapy. In order for a new service to manage patients with late GI effects of pelvic radiotherapy successfully, engagement and enthusiasm from both local oncology and gastroenterology services is highly important. Long-term sustainability (ie, the ability to move from short-term research funding into long-term service provision) is also a hurdle that must be overcome.

\section{METHODS AND ANALYSIS \\ Aims and objectives}

The aim of this study is to implement successfully an innovative service that aims to improve the care offered to men diagnosed with prostate cancer, and also to their partners and families, in a postcurative treatment setting at three sites.

The specific objectives are:

- To raise awareness and increase expert capacity for the treatment of GI late effects following radiotherapy;

- To implement a tested interventional model of care in order to develop centres of excellence that are specifically focused on the early identification and management of GI late effects;

- To evaluate the effectiveness of the new service via healthcare usage analysis, health economics, quality of life and by assessing the acceptability to healthcare professionals (HCP) and patients and families;

- To monitor the experiences of patients, support givers and members of staff regarding service implementation. The specific objectives here are:

- To investigate the barriers to setting up a new service in local practice;

- To evaluate solutions to those barriers; 
- To assess the effectiveness of training in relation to safe and independent coordination of services.

\section{The intervention}

The proposed intervention (outlined in figure 1) is a package of multidisciplinary care aimed at the early identification and the subsequent intervention to reduce the physical, psychological and social impact of late GI effects after curative treatment for prostate cancer.

\section{Study design}

The Evaluating and Addressing the Gastrointestinal Late Effects (EAGLE) study is a mixed methods implementation study. The study involves the development of a new package of enhanced assessment, multidisciplinary care and use of treatment algorithms will be assessed within an implementation-research framework. Implementation research can be defined as 'the scientific inquiry into questions concerning implementation-the act of carrying an intention into effect, which in health research can be policies, programmes or individual practices (collectively called interventions) ${ }^{15}$ The success of the implementation is based on real-world outcomes; acceptability; adoption; appropriateness; feasibility; fidelity; implementation cost; incremental cost of intervention delivery and costs to other NHS services; coverage; sustainability. ${ }^{16}$

Outcomes will be measured via collection and analysis of both quantitative and qualitative data sets. Mixed

The intervention to be implemented comprises:

1. DESIGNER assessment toolo-: to be used by oncology/ urology teams to identify patients at risk/ suffering from GI "late" effects following pelvic radiotherapy. The DESIGNER tool consists of three questions that aim to identify patients suffering from $\mathrm{Gl}$ symptoms following pelvic radiotherapy. The questions cover faecal incontinence, rectal bleeding and impact on quality of life. Patients are asked to answer 'yes' or 'no' to each question. Any patient who answer 'yes' requires a referral to a gastroenterologist (appendix 1);

2. Rapid referral: to specialist clinic staff, such as specially trained clinical nurse specialists (CNS)/dieticians/junior doctors with back-up from specialist consultant gastroenterologists;

3. In-depth patient assessment: holistic and GI focused patient assessments will be carried out by the specialist clinic staff;

4. Treatment algorithm: Current version of gold standard treatment protocols (Guide to Managing Gastrointestinal Symptoms of Pelvic Radiation Disease) $)^{26}$;

5. Standardised training and ongoing expert support (see section 7.1)

6. Data sharing: Standardised patient assessment and reported outcomes, enabling data sharing across centres;

7. Assessment of the benefit provided by the new intervention

Figure 1 Summary of the intervention. The figure offers a breakdown of the gastrointestinal intervention provided as part of the study. Patients will be screened for late gastrointestinal effects in oncology using a simple screening tool,

ALERT-B. Patients with symptoms will then be referred to gastroenterology to a specialist team who will follow an algorithm to offer targeted investigations and treatment. ALERT-B, Assessment of Late Effects of

RadioTherapy-Bowel; EAGLE, Evaluating and Addressing the Gastrointestinal Late Effects study; GI, gastrointestinal. methods are suitable for implementation research as they provide a practical way to understand multiple perspectives and outcomes. ${ }^{15}$ This approach will involve a quantitative before and after (treatment) study, as well as a substudy involving the use of a comparison group and a qualitative study.

This study will use an overarching framework suited to complex interventions called normalisation process theory (NPT). ${ }^{17}$ NPT is a pragmatic theory that examines the factors needed for successful implementation into local practice. NPT allows one to examine those factors that are essential for new initiatives in healthcare settings where multiple stakeholders' perspectives and resource availability can 'sink' new developments or can undermine trials. ${ }^{18}$ Murray et $a l^{18}$ and Meyer et $a l^{19}$ discussed the role of NPT in testing the feasibility, adherence and acceptance of initiatives. These analyses were coupled with an evaluation of cost and sustainability per site, albeit with scope for local idiosyncrasies.

\section{Implementation centres}

This study will be carried out in three centres across the UK. Sites will be invited to complete a template of existing services to apply for selection to the study (see online supplementary file 1 for an example of the site information form).

Criteria for site selection:

- Clinical oncology team (from the same catchment area as the gastroenterology team) managing around 100 patients per year with prostate cancer who have been treated with radical radiotherapy.

- Ability to identify a prostate-cancer oncologist willing to use the Assessment of Late Effects of RadioTherapy-Bowel (ALERT-B) screening tool to identify and recruit patients for the EAGLE study.

- Ability to identify a gastroenterologist who is willing to:

- Support the introduction of a non-consultant post following the 'Guide to Managing Gastrointestinal Symptoms of Pelvic Radiation Disease for investigation and management of pelvic radiation disease', $;^{20}$

- Provide ongoing clinical support to the nonconsultant postholder;

- Adhere to the RMH algorithm;

- Lead the local process for securing sustainable funding.

- Additional key considerations include ongoing local developments in this field including involvement of oncologists managing patients with other cancers who are known to suffer pelvic radiation disease (PRD).

\section{Enhanced staff training}

Training options for the specialist PRD team will include completion of the Macmillan Cancer Support-RMH online training module, teaching and support sessions with the RMH nurse consultant, and visits to the RMH PRD multidisciplinary clinic to gain experience of how 
the PRD service is delivered at the RMH. The GI and nutrition team at the RMH have developed a robust teaching package and supportive documentation for new units. This teaching programme is supported by an online validated BSc/MSc module run by The Royal Marsden School. This training package will be aimed at both the specialist PRD team and the wider nonspecialist oncological and gastroenterological teams. Once a service is established, intermittent multidisciplinary update sessions (at least annually) will be provided to ensure that skills are maintained. These update sessions will be provided within EAGLE by ongoing support from the RMH PRD multidisciplinary team (MDT) for the duration of the study.

\section{Enhanced MDT care}

A streamlined referral pathway to a named healthcare professionals in the new multidisciplinary PRD service in each centre will be established for EAGLE study participants who are identified with late GI effects of pelvic radiotherapy. The referral will include relevant oncology and radiotherapy treatment information. During the first appointment at the new gastroenterology clinic, a comprehensive medical history will be taken. Detailed gastroenterological status will be ascertained by following the $\mathrm{RMH}$ algorithm, which will be used to arrange appropriate investigations. ${ }^{9} \mathrm{~A}$ list of investigations can be found in online supplementary file 2 .

Follow-up investigations will be arranged by telephone or clinic visits as necessary, although it is envisaged that patients will be seen in clinic at a minimum of 6 and 12 months after initial assessment. Staffing, coordination and running of enhanced services will be agreed on a site-by-site basis, according to local preferences. The research team will liaise with these sites to create protocols for practice that are agreed locally, as required.

\section{Participant selection and recruitment}

Establishing patient eligibility will be a two-stage process. Initially, those patients attending urology/oncology follow-up clinics that received radiotherapy treatment for prostate cancer at least 6 months previously will be asked to consent to the registration phase of EAGLE by a Good Clinical Practice (GCP) trained healthcare professional working at the oncology clinic.

Those patients who consent will be screened for eligibility by completing the ALERT-B tool. The ALERT-B tool (see online supplementary file 3 ) has been developed and tested for content and face validity, ${ }^{21}$ by members of the EAGLE research team previously as part of a Tenovus Innovation Grant. As part of the EAGLE study, the ALERT-B tool will also be validated in terms of reliability against the Gastrointestinal Symptom Rating Score (GSRS).

The ALERT-B tool will be reviewed with the patient by the clinical nurse specialist (CNS), research nurse, research radiographer or doctor. Those patients with at least one 'yes' response to one of the three questions (experiencing faecal incontinence, rectal bleeding or significant impact on quality of life) will be identified to the consultant oncologist and they will be registered for the EAGLE study. Patients will be offered the choice to be referred to the multidisciplinary pelvic disease clinic and they will be given written information about the EAGLE study.

At both stages, patients will be excluded if they meet one or more of the following criteria: any factor that affects their ability to communicate their wishes; an inability to comprehend what is being asked of them; a lack of capacity to consent; or, cancer recurrence.

To benefit the maximum number of men, patients will be recruited until the local gastroenterology service capacity is reached. It is estimated that 100 patients from each centre will be screened annually and those patients who are referred will be managed within the service for 12 months with an average of three appointments during this time. Long-term, the expectation is that a full-time CNS would manage $\sim 150$ new patients per year.

\section{Participants for interviews}

Participants will be sampled purposively from the three research sites across three groups to represent:

1. Oncology and GI HCPs in order to capture staff attitudes to implementation;

2. Patients receiving services in order to capture patient experience of services;

3. Support givers to the family in order to represent family or caregiver perspectives of services.

The study aims to recruit 10-15 participants from each of the three groups listed above. Participants will be interviewed at three time points over the course of 8 14 months. Interviews will also be conducted with key informants on a local basis, where appropriate. For example, these informants include primary care providers and other stakeholders including Macmillan Cancer Support services, commissioners and Local Health Board representatives.

Participants must be aged 18 years and over and they must be able to undertake an interview in English without the need for translation (unless local translation services are readily available) in order to be eligible for inclusion in the study. All those participants who are eligible will be asked to participate until enough participants have been recruited to represent each group (ie, until 'saturation') and to allow for follow-up interviews. Participants will be interviewed by either a face-to-face interview at a location of their choice or over the telephone. Interviews will last $\sim 30-60 \mathrm{~min}$. The interview will be terminated earlier if a participant is thought to be fatigued, upset or becomes unwell. In the event of participant distress due to discussion of sensitive topics, or if a clinical or work-related issue emerges, the researchers, will react at the time according to their own experience. The researchers will then refer the issue to the participant's clinical team (with the participant's permission). 


\section{Data collection}

Data will be collected at baseline, 6 months ( \pm 2 months) and 12 months ( \pm 2 months) after entry into the study by a member of the research team. The diagram below (figure 2) demonstrates the pathway that EAGLE participants will follow and the data collection instruments that will be used in the study. A case report form for each assessment point will be assigned to each participant in order to collect questionnaire and relevant clinical data.

There are multiple (end point) domains to be considered including: physical; psychological; sexual; financial; and, social domains. There is some level of interdependence and overlap between these domains. The study will focus initially on physical effects, which can be broadly separated into gastrointestinal, genitourinary, sexual, endocrinal and fatigue. The study end points are designed to capture acceptability and effectiveness of the intervention and are as follows:

- Bowel-specific health-related quality of life (HRQoL);

- Global HRQoL;

- Prostate-specific HRQoL;

- Patient, support giver and staff experience;

- Healthcare resource usage;

- Cost and acceptability of staff training.

\section{Bowel-specific HRQoL}

The GSRS is a validated questionnaire addressing symptoms that are important and relevant to patients with general GI symptoms. GSRS evaluates the absence or presence (and severity) of 15 GI symptoms using a fourpoint response scale over a 1-week period prior to administration. GSRS assesses five domains that have been identified as important to GI integrity: reflux syndrome; acute pain syndrome; indigestion syndrome; diarrhoea syndrome; and, constipation syndrome. GSRS has a range of scores going from zero to three (zero representing good health and three poor health). This questionnaire was chosen for use in this study because its reliability and validity are well-documented and normal values for a general population are available. ${ }^{22}$

\section{Global HRQoL}

Global HRQoL will be measured using the EQ-5D-5L and the European Organisation for Research and Treatment of Cancer Quality of Life Core Questionnaire 30 (EORTC QLQ C30).

A newly released validated version of the EQ-5D-5L with five response categories (mobility, self-care, usual activities, pain/discomfort and anxiety/depression) for each dimension provides enhanced discriminatory power will be used in the study. ${ }^{23}$ This tool also measures HRQoL using a vertical visual analogue scale. ${ }^{24}$

The 30-item questionnaire incorporates five functional scales and is a reliable and clinically valid measure of quality of life in patients with cancer. ${ }^{25}$ Participants will be asked to complete a number of questionnaires, and so to limit the burden of this process they will be required to answer only questions 29 and 30 (ie, items that rate overall global health and quality of life) from the EORTC QLQ C30 questionnaire. Item responses to these two questions from the EORTC QLQ C30 questionnaire are numerical scales going from 1 to 7 . The scores from both items are added together and their sum is transformed linearly to lie in the range $0-100$, where 0 represents the worst possible global HRQoL and 100 represents the best possible global HRQoL.

\section{Prostate-specific HRQoL}

Prostate-specific HRQoL will be measured using the European Organisation for Research and Treatment of Cancer Quality of Life Questionnaire Prostate Cancer module (EORTC QLQ-PR25) and the Expanded Prostate Cancer Index Composite (EPIC-26). Both prostate questionnaires were requested by the funder, Prostate Cancer UK (PCUK), to collect data as part of a series of global projects.

The EORTC QLQ-PR25 questionnaire contains 25 items that are grouped into 5 multi-item scales. High scores reflect either more symptoms (urinary, bowel, hormonal treatment-related symptoms) or high levels of functioning (sexual). ${ }^{26}$ All items and scale scores of the EORTC QLQ-PR25 are transferred linearly onto a 0-100 scale, with higher scores again reflecting either more symptoms (urinary, bowel, hormonal treatment-related symptoms) or higher levels of functioning (sexual). ${ }^{24} \mathrm{~A}$ shorter 26-item version of EPIC was designed and validated. ${ }^{27}$ EPIC-26 has shown satisfactory test-retest reliability and internal consistency for the urinary, bowel, sexual and hormonal domains. ${ }^{28}$

\section{Acceptability}

Experience of the new service will be explored via longitudinal semistructured interviews with participants. The data obtained from these interviews will be used to highlight problems or to inform necessary adjustments of GI symptom screening and the new gastroenterology service. In addition interviews with participants and support givers will provide an insight into the psychological and social impact of GI symptoms and the new GI service. At the time of completion of the study, an analysis will be carried out of all of the data sets in order to report common themes and differences across participant groups and settings. This analysis will contribute to a model of implementation for future sites in the UK.

\section{Health resource usage}

Resources used to deliver the intervention in all of the centres will be monitored prospectively, including those resources used in training staff, screening patients and all patient contact, investigations, etc. Research costs will be isolated and they will not be included in the costing.

Resource usage data will be collected via two bespoke resource-use questionnaires. One questionnaire will be completed by all participants and the other questionnaire will be completed by designated health 
professionals. Both questionnaires will be administered at baseline and at each follow-up point. Patient recall has been shown to be a valid method for collecting NHS resource-use data. ${ }^{29}$

\section{Comparative patient data}

Comparative patient subsequent resource use data will be collected from a Cardiff subgroup. Participants in the substudy will be managed following current standard care, either within the Velindre Cancer Centre or with referral to gastroenterology services in Cwm Taf. Data from this group will help to compare the impact of the new GI service against standard care. These patients will be given information on the substudy, which will involve an assessment of their healthcare resource usage and health-related quality of life over a 12-month period from study entry. The EQ-5D-5L and EPIC-26 will also be completed at each data collection point.

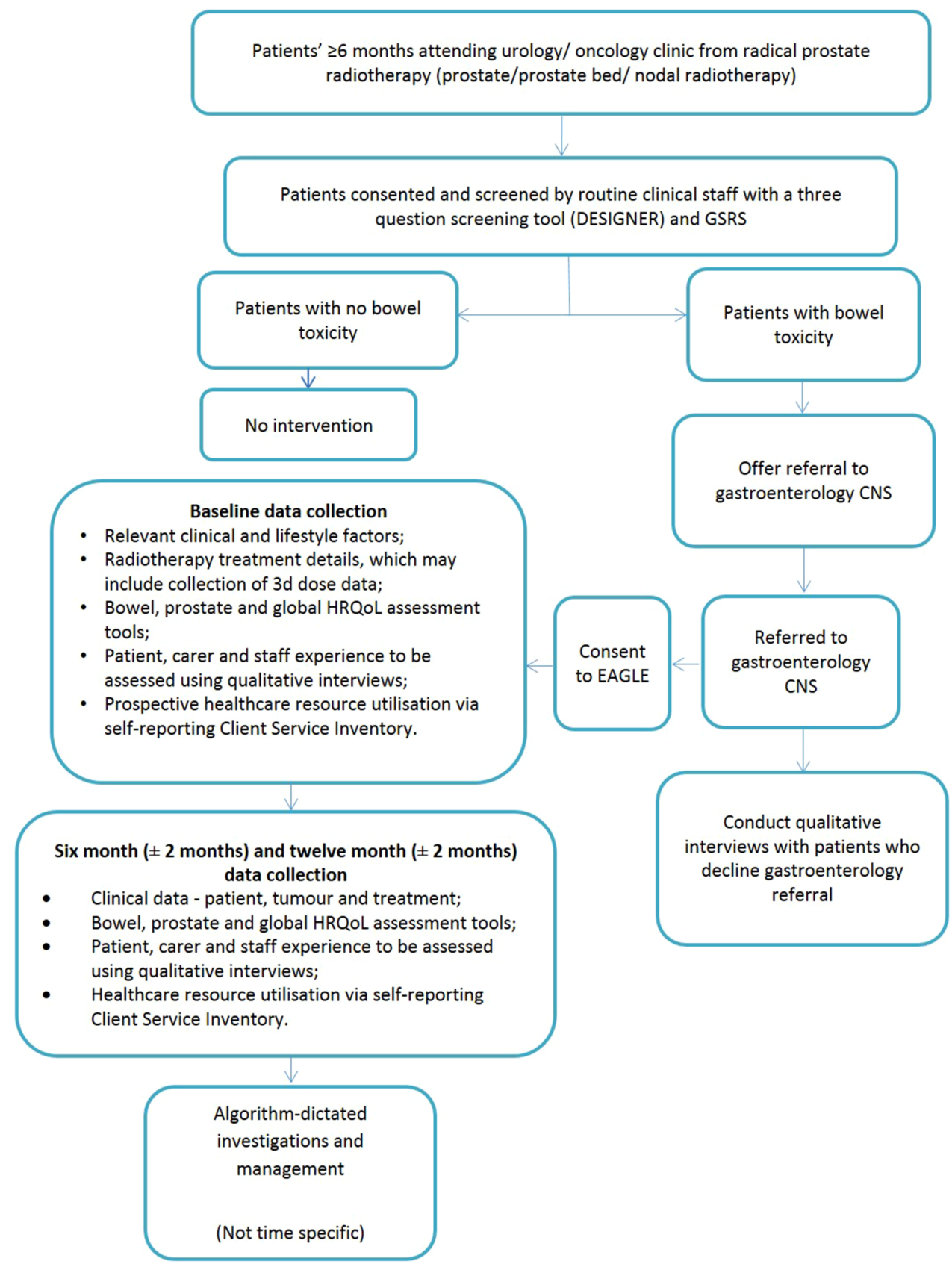

Figure 2 EAGLE study Pathway. The figure provides an overview of the study processes and data collection at different stages of the EAGLE study. Participants will consent to be screened for the late gastrointestinal effects of radiotherapy at their routine oncology appointments. Only patients identified with bowel symptoms will be offered a referral to gastroenterology where consent will be taken for questionnaire data collection at three time points (baseline, around 4-8 months and 10-14 months). ALRT-B, Assessment of Late Effects of RadioTherapy-Bowel; CNS, clinical nurse specialist; EAGLE, Evaluating and Addressing the Gastrointestinal Late Effects study; HRQoL, health-related quality of life; GSRS, Gastrointestinal Symptom Rating Score. 


\section{Data analysis}

Participant and treatment characteristics will be explored by using descriptive statistics. Measures of centre (mean and median) and measures of spread (SD and IQR) will be used to characterise outcome measures. Subscale, and any appropriate overall scores, will be found according to relevant scoring manuals (eg, for EORTC QLQ-C30 ${ }^{30}$ or EPIC ${ }^{31}$ ) and with the use of any pre-existing and tested software (eg, in R using the preexisting package 'QoLR' for EORTC QLQC30 and PR25). Improvement in outcomes will be reported by comparing the 6 and 12-month follow-up measures to the baseline measurements and to the shadow comparison group. Effect sizes such as standardised means from pretreatment to post-treatment (for 6 months post via, eg, (mean baseline-mean 6 post)/SD baseline) will be used to characterise these improvements. Standard methods for comparing paired or repeated-measures data will be employed in order to test if improvements are statistically significant at the $5 \%$ level (eg, paired tests and repeated-measures analysis on variance using parametric or non-parametric methods, as appropriate). All participants will be encouraged to complete all of the questionnaires at all of the time points, and so it is imagined that complete-case analysis can be employed.

A generic procedure will be used for missing data that forms subscale scores from those items (appropriately weighted) in a subscale that are non-missing, if no more than $50 \%$ of items in the subscale are missing. If more than $50 \%$ of items are missing then the subscale score is also set to be missing. This process accounts for 'itemwise' missing data when forming subscale scores for each patient, although it does not account for any attrition of patients during the study. If attrition from the study does occur (eg, through cancer recurrence through the course of the intervention, unacceptability of the intervention to some men, or burden of the research) then appropriate statistical modelling techniques that account for this effect and/or the use of imputation techniques (eg, multiple imputation) can be employed.

\section{Cost-effectiveness analysis}

A cost-effectiveness analysis in the form of a cost utility analysis, comparing participants in the EAGLE study with those not receiving the service will be undertaken from an NHS perspective for the primary analysis. Total costs (intervention plus or minus any subsequent differences in NHS costs) will be assessed against effects in terms of quality adjusted life years (QALY) based on the EQ-5D-5L. The estimated incremental cost per QALY from the service can be compared with the willingness to pay threshold of $£ 20000-£ 30000$ per extra QALY currently used by The National Institute for Health and Care Excellence (NICE) ${ }^{32}$

Secondary analysis will take a wider perspective and will include missed workdays and patient-borne costs (including out-of-pocket expenses relating to travel, carer provision and incontinence products). The sensitivity of the conclusions to changes in assumptions will be assessed via sensitivity analysis.

\section{Costing staff training}

Professionals delivering the intervention will be asked to complete a training record on a weekly basis throughout the duration of the research study. This record will facilitate measurement of training costs, which will feed into the cost-effectiveness analysis. All formal training, in addition to more ad hoc training and support from dieticians, consultants or other healthcare professionals, will be logged regardless of whether it is received during or outside of working hours.

\section{Interview data analysis}

The researcher will audio record the interview digitally. Interviews will be transcribed verbatim and the interview data will be uploaded to qualitative analysis software called QSR NVivo V.10. Ten per cent of transcripts will be co-coded by a senior researcher as a measure of reliability. Each interview will be read initially with a deductive approach to isolate significant problem areas in the implementation delivery. The anonymised data will be represented by selected extracts in a narrative format with a thematic structure.

Data will be analysed in order to identify problems with local systems and procedures. This analysis will also explore the HCP and patient and support giver attitudes and experiences. Framework analysis is the most appropriate analytic method to achieve these aims for the interview data. ${ }^{27}$ Framework analysis is suited to applied health research in which a certain amount of existing knowledge is available and where the aim of the study is to inform future practice rather than theoretical development. $^{33}$ Framework analysis involves a systematic process of sifting, charting and sorting the material into key issues and themes'. ${ }^{27}$ It allows the integration of a priori issues into the emerging data analysis and it provides a clearly defined analytical structure that contributes to the transparency and validity of the results. The matrix for the framework analysis will be developed from a deductive approach using NPT, further supported by an overall inductive analysis of spontaneously arising themes during interviews. ${ }^{34}$

\section{Project management}

A study management group (SMG) will be responsible for the day-to-day management of the study and they will meet regularly to advise on the promotion and running of the study. SMG members will include the chief investigator (CI), coinvestigators, project staff and patient representatives. Members will sign a SMG charter outlining their roles. The study will be coordinated through the Marie Curie Palliative Care Research Centre. 
Consent

Signed consent will be taken at least 24 hours after the patient has been given the relevant participant information sheet.

A separate information and consent process to the main study will be used for interviews as this is a subset of the main study. The principal investigator or clinical team at each site will identify and discuss the interview substudy with eligible patients, support givers and HCPs before consent can be obtained. Similarly, the participant will remain free to withdraw at any time from the study. All data will be withdrawn at request.

\section{Confidentiality and data management}

The CI and the research team of EAGLE will preserve the confidentiality of participants in accordance with the Data Protection Act $1998 .^{35}$ Data will be stored on a password-protected computer located in secure University buildings and appropriately backed up. All data will be retained for up to 15 years after the closure of the study.

\section{ETHICS AND DISSEMINATION}

\section{Ethical issues}

Currently only a small proportion of patients are referred to a gastroenterologist for further investigation into bowel symptoms that develop after pelvic radiotherapy. This may be because patients feel uncomfortable discussing their bowel symptoms with healthcare professionals. ${ }^{10}$ The SMG will draw on their considerable experience in conducting research in this area to ensure a design sensitive to this patient group.

Research data and patient-related information will be managed in accordance with relevant regulatory approvals. The study has been adopted onto the UK Clinical Research Network (UKCRN) portfolio under ID number 16974. In addition the study is sponsored by Cardiff University (SPON1238-14).

\section{Public and patient involvement}

Public and patient involvement has been integral to the conduct of this study from the initial funding application. The SMG includes two research partners who have personal experience of the effect that late GI effects of pelvic radiotherapy can have on the patient and their family and friends. The research partners provide a valuable source of experience that has particularly helped when drafting appropriate participant-facing study documents. Members of the research team will work alongside the research partners to identify and address specific support or training requirements to enable their effective participation.

\section{Methods of disseminating the findings}

Multiple methods of disseminating the findings will include a written report for the study funders, PC UK, including an executive summary with clearly identified practice suggestions to benefit patients, support givers and HCPs. The study will adhere to PCUK data sharing agreement with the National Cancer Research Institute (NCRI). As PCUK is a member of the NCRI, an agreement is in place to share anonymous data on the research portfolio with the International Cancer Research Partnership (ICRP). Papers detailing the results of this study will be submitted for publication in peer-reviewed journals and at national and international conferences.

Participants (or their families) will be informed on request about the progress of the study. Summary reports will be displayed on the website, although patients or their families will be able to request hard copies if they prefer. For reasons of confidentiality, original data (audio recordings) and transcripts will not be shared.

\section{Author affiliations}

${ }^{1}$ Marie Curie Palliative Care Research Centre, School of Medicine, Cardiff University, Cardiff, UK

${ }^{2}$ Specialist Unit for Review Evidence, Cardiff University, Cardiff, UK

${ }^{3}$ Gastroenterology Unit, Royal Marsden Hospital, London, UK

${ }^{4}$ Department of Oncology, Weston Park Hospital, Sheffield Teaching Hospitals NHS Foundation Trust, Sheffield, UK

${ }^{5}$ School of Dentistry, Cardiff University, Cardiff, UK

${ }^{6}$ Faculty of Life Sciences and Education, Health Economics and Policy

Research Unit (HEPRU), University of South Wales, Cardiff, UK

${ }^{7}$ Department of Gastroenterology, University Hospital of Llandough, Cardiff, UK

${ }^{8}$ Macmillan Cancer Support, London, UK

${ }^{9}$ Department of Oncology, University of Sheffield, Sheffield, UK

${ }^{10}$ Swansea Centre for Health Economics, College of Human and Health

Sciences, Swansea University, Swansea, UK

${ }^{11}$ Velindre Cancer Centre, Cardiff, UK

${ }^{12}$ Institute of Cancer and Genetics, Cardiff University, Cardiff, UK

Twitter Follow Annmarie Nelson at @annmarie0

Contributors JS and AN are responsible for the overall design of the study. JA, JG, AM, SA, CF, DC, LS and SP are the coinvestigators. ST, DJJF and WD drafted the manuscript. All authors provided input into the protocol, critical feedback on the manuscript and approved the final manuscript. The study sponsor and funder were not involved in the study design or writing of the protocol.

Funding This work is supported by Prostate Cancer UK's True NTH initiative. The funder reference number is $250-55$. AN's post is fully supported by Marie Curie Cancer Care core grant funding, grant MCCC-FCO-14-C.

Competing interests None declared.

Patient consent Obtained.

Ethics approval This study has gained approval from NHS Research Ethics Committee (REC) NRES Committee North West-Liverpool East REC Reference -14/NW/1206 and NHS Research and Development (NHS R\&D) approval from the sites.

Provenance and peer review Not commissioned; externally peer reviewed.

Open Access This is an Open Access article distributed in accordance with the Creative Commons Attribution Non Commercial (CC BY-NC 4.0) license, which permits others to distribute, remix, adapt, build upon this work noncommercially, and license their derivative works on different terms, provided the original work is properly cited and the use is non-commercial. See: http:// creativecommons.org/licenses/by-nc/4.0/

\section{REFERENCES}

1. UK CR. Prostate Cancer Statistics. Secondary Prostate Cancer Statistics 2013. February 2016. http://www.cancerresearchuk.org/ health-professional/prostate-cancer-statistics 
2. Institute NC. Common Terminology Criteria for Adverse Events. Secondary Common Terminology Criteria for Adverse Events. 2015 http://ctep.cancer.gov/protocolDevelopment/electronic_applications/ ctc.htm\#ctc 4016th February 2016.

3. Peschel RE, Colberg JW. Surgery, brachytherapy, and external-beam radiotherapy for early prostate cancer. Lancet Oncol 2003;4:233-41.

4. Wagner W, Bölling T, Hambruegge C, et al. Patients' satisfaction with different modalities of prostate cancer therapy-a retrospective survey among 634 patients. Anticancer Res 2011;31:3903-8.

5. Gami B, Harrington K, Blake P, et al. How patients manage gastrointestinal symptoms after pelvic radiotherapy. Aliment Pharmacol Ther 2003;18:987-94.

6. Henson CC, Andreyev HJ, Symonds RP, et al. Late-onset bowel dysfunction after pelvic radiotherapy: a National survey of current practice and opinions of clinical oncologists. Clin Oncol (R Coll Radiol) 2011;23:552-7.

7. Andreyev J. Gastrointestinal symptoms after pelvic radiotherapy: a new understanding to improve management of symptomatic patients. Lancet Oncol 2007;8:1007-17.

8. Resnick MJ, Koyama T, Fan KH, et al. Long-term functional outcomes after treatment for localized prostate cancer. N Engl J Med 2013;368:436-45.

9. Andreyev HJN, Benton BE, Lalji A, et al. Algorithm-based management of patients with gastrointestinal symptoms in patients after pelvic radiation treatment (ORBIT): a randomised controlled trial. Lancet 2014;382:2084-92.

10. Andreyev HJ. Gastrointestinal problems after pelvic radiotherapy: the past, the present and the future. Clin Oncol (R Coll Radiol) 2007;19:790-99.

11. Andreyev J. Gastrointestinal complications of pelvic radiotherapy: are they of any importance. Gut 2005;54:1051-4.

12. McClelland S. Health policy in Wales-distinctive or derivative? Social Policy and Society 2002;1:325-33.

13. Henson CC, Davidson SE, Lalji A, et al. Gastrointestinal symptoms after pelvic radiotherapy: a national survey of gastroenterologists. Support Care Cancer 2012;20:2129-39.

14. Andreyev HJN, Vlavianos P, Blake P, et al. Gastrointestinal symptoms after pelvic radiotherapy: role for the gastroenterologist? Int J Radiat Oncol Biol Phys 2005;62:1464-71.

15. Peters DH, Adam T, Alonge O, et al. Republished research: implementation research: what it is and how to do it implementation research is a growing but not well understood field of health research that can contribute to more effective public health and clinical policies and programmes. This article provides a broad definition of implementation research and outlines key principles for how to do it. Br J Sports Med 2014;48:731-6.

16. Proctor E, Silmere H, Raghavan R, et al. Outcomes for implementation research: conceptual distinctions, measurement challenges, and research agenda. Adm Policy Ment Health 2011;38:65-76

17. May CR, Mair F, Finch T, et al. Development of a theory of implementation and integration: normalization process theory. Implement Sci 2009;4:29.

18. Murray E, Treweek S, Pope C, et al. Normalisation process theory: a framework for developing, evaluating and implementing complex interventions. BMC Med 2010;8:63.
19. Meyers DC, Durlak JA, Wandersman A. The quality implementation framework: a synthesis of critical steps in the implementation process. Am J Community Psychol 2012;50:462-80.

20. Andreyev HJN, Muls AC, Norton C, et al. Guidance: the practical management of the gastrointestinal symptoms of pelvic radiation disease. Frontline Gastroenterology 2015;6:53-72.

21. Rattray J, Jones MC. Essential elements of questionnaire design and development. J Clin Nurs 2007;16:234-43.

22. Svedlund J, Sjödin I, Dotevall G. GSRS-a clinical rating scale for gastrointestinal symptoms in patients with irritable bowel syndrome and peptic ulcer disease. Dig Dis Sci 1988;33:129-34.

23. Dimenäs E, Glise H, Hallerbäck B, et al. Quality of life in patients with upper gastrointestinal symptoms: an improved evaluation of treatment regimens? Scand J Gastroenterol 1993;28:681-7.

24. Janssen MF, Pickard AS, Golicki D, et al. Measurement properties of the EQ-5D-5L compared to the EQ-5D-3L across eight patient groups: a multi-country study. Qual Life Res 2013;22:1717-27.

25. Aaronson NK, Ahmedzai S, Bergman B, et al. The European Organization for Research and Treatment of Cancer QLQ-C30: a quality-of-life instrument for use in international clinical trials in oncology. J Natl Cancer Inst 1993;85:365-76.

26. van Andel G, Bottomley A, Fosså SD, et al. An international field study of the EORTC QLQ-PR25: a questionnaire for assessing the health-related quality of life of patients with prostate cancer. Eur $J$ Cancer 2008;44:2418-24.

27. Szymanski KM, Wei JT, Dunn RL, et al. Development and validation of an abbreviated version of the expanded prostate cancer index composite instrument for measuring health-related quality of life among prostate cancer survivors. Urology 2010;76:1245-50.

28. Wei JT, Dunn RL, Litwin MS, et al. Development and validation of the expanded prostate cancer index composite (EPIC) for comprehensive assessment of health-related quality of life in men with prostate cancer. Urology 2000;56:899-905.

29. Thorn JC, Coast J, Cohen D, et al. Resource-use measurement based on patient recall: issues and challenges for economic evaluation. Appl Health Econ Health Policy 2013;11:155-61.

30. Fayers PM, Aaronson NK, Bjordal K, et al. On behalf of the EORTC Quality of Life Group. EORTC QLQ-C30 scoring manual: EORTC, Brussels, 1999

31. Sanda M, Wei J, Litwin M. Scoring Instructions for the Expanded Prostate cancer Index Composite Short Form (EPIC-26) https:// medicine.umich.edu/sites/default/files/content/downloads/Scoring\% 20Instructions\%20for\%20the\%20EPIC\%2026.pdf (accessed 1 Mar 2016).

32. National Institute for Health and Care Excellence. Guide to the methods of technology appraisal Secondary Guide to the methods of technology appraisal. 2013. https://www.nice.org.uk/article/pmg9/ chapter/forewordFebruary2016

33. Ritchie J, Spencer L. Qualitative data analysis for applied policy research. The Qualitative Researcher's Companion 2002:305-29.

34. May C, Murray E, Finch T, et al. Normalization process theory online users' manual and toolkit. NPT: Normalization Process Theory 2010. http: www normalizationprocess org (accessed 12 Mar 2012).

35. Kingdom U. Data Protection Act 1998. http://www.legislation.gov.uk/ ukpga/1998/29/contents (accessed 1 Mar 2016). 